\title{
Valorization of Tomato Processing Waste for Lycopene Extraction
}

\author{
Rim Driouich*, Olfa Bousselmi and Ameur Cherif \\ Laboratory of Biotechnology and Valorization of Bio-Geo Resources \\ BVBGR LR11ES31 \\ Institut Supérieur de Biotechnologie de SidiThabet \\ University of Manouba, BioTechPole Sidi Thabet 2020 Sidi Thabet, Ariana, Tunisia
}

\begin{abstract}
Lycopene is one of the most powerful antioxidants and singlet oxygen quenching agents. It has been found to be of great medical importance with various anti cancer effects, cardiovascular diseases effective agent and with its ability to be an important carotenoid in preventive medicine. In Tunisia, we note a real consumption of tomato paste. With 28 production units for double and triple concentrated tomato, noting a significant production of waste (approximately 30000 Tonne/year) consisting of skins pulp and seeds, which could be an important source of lycopene. Our study is concerned with three solid-phase extraction process: assisted by ultrasonic, by microwave and by homogenizer heating at moderate temperature. Their efficiency for the extraction of lycopene contained in the tomato waste was elaborated. The effect of the main process variables (including solvent polarity, solid-to-liquid ratio, temperature and time) on lycopene recovery have been investigated. The yield of the isolated lycopene was determined using UV-Visible spectrophotometry and UPLC-DAD quantification methods. Lycopene-rich extract was obtained from the dried and milled tomatoes waste using ethanol as a food grade extraction solvent and employing microwave assisted extraction process. The optimum conditions were $30 \mathrm{~min}$ total extraction time at $50^{\circ} \mathrm{C}$ temperature and $1 \mathrm{bar}$ pressure. The degradation of extracted lycopene through air oxidation and day light effect were also tested. Lycopene is stable when mixed with olive oil as a natural antioxidant. Up to $80 \%$ of lycopene degradated when exposed for one month to day light at $25^{\circ} \mathrm{C}$.
\end{abstract}

Keywords : Lycopene, tomatoes, extraction, microwave, plants waste valorization.

\section{Introduction}

By-products of fruits and vegetables processing represent a major disposal problem for the industry concerned, but they are also promising sources of compounds which may be used for various purposes in food, pharmaceutical and cosmetic industries ${ }^{1-2}$.

Tomato (Lycopersiconesculentum) is, after potato, the second most consumed vegetable in the world and approximately $30 \%$ is consumed as processed products. Both fresh and processed tomato contain a high nutritional value, due to its content of different types of micronutrients: carotenoids (lycopene and $\beta$-carotene), vitamins ( $\mathrm{C}$ and $\mathrm{E}$ ), folates, and polyphenolic compounds (flavonoids and phenolic acids) ${ }^{3-4}$. The skin of tomatoes has been found to be richer sources of lycopene than the water insoluble fraction and the fibrous fraction ${ }^{5}$. However, when tomatoes are processed into products, $3-7 \%$ of their weight becomes waste ${ }^{6-7}$. Tomato waste, known as tomato pomace, contains a

*Corresponding author: Rim Driouich

Email address: dodrim@gmail.com

DOI: http://dx.doi.org/10.13171/mjc61/0161107123915/driouich significant amount of the fruit peels, which has no commercial value and is currently disposed as a solid waste or used for animal feeding. Nevertheless, a careful examination of this material reveals that it is a rich source of important nutrients and phytochemicals that could provide a potential source of natural lycopene.

Lycopene is an acyclic tetraterpenic hydrocarbon with 13 carbon-carbon double bonds, 11 of which are conjugated, which has attracted a great attention in recent years for its potential health benefits ${ }^{8}$. The high degree of conjugation makes this carotenoid one of the most potent natural antioxidants, with a singlet-oxygen quenching ability twice as high as that of $\beta$-carotene and ten times higher than that of $\alpha$ tocopherol ${ }^{9}$. Moreover, its activity is synergistically enhanced by the presence of other tomato phytochemicals including $\beta$-carotene, phytoene and phytofluene ${ }^{10}$. These properties are thought to be responsible for the apparent inverse association between the consumption of lycopene-rich foods

Received September $2^{\text {nd }}, 2016$

Accepted October $21^{\text {st }}, 2016$

Published November $7^{\text {th }}, 2016$ 
and the incidence of cardiovascular disease and cancer $^{11-12}$.

Advanced valorisation strategies based on green chemical technologies are more appealing from both, the practical-economic and the sustainability points of view, using combined bio/chemo-technological protocols ${ }^{13}$.

The extraction, handling and analysis of lycopene must be carried out under controlled environmental conditions to minimize oxidative degradation and isomer formation. Several extraction technologies by either conventional ${ }^{14}$, or supercritical fluid 15-16 were reported, but the obtained yield did not exeed $7,19 \mu \mathrm{g} / \mathrm{g}$. Supercritical fluid with central composite rotatable design model was proposed by Kassama et al. ${ }^{17}$, the recovery of all lycopene was 3,3\%. Supercritical carbon dioxide extraction technology was also reported by Topal et al. ${ }^{18}$, the maximum reached yield of lycopene in tomato skin sample was $1,18 \mathrm{mg} / \mathrm{g}$. Furthermore, Nobre et al. ${ }^{19}$ extracted lycopene from tomato industrial wastes by supercritical extraction with ethane, which seems to be a better solvent than supercritical $\mathrm{CO}_{2}$ leading to faster extraction with a little better recovery. However supercritical fluid technology is considered as a developped alternative, with some limits dealing with time and cost consuming.

Moreover, enzymatic Assisted extraction has been also reported ${ }^{20-21}$, Choudhari et al. increased the yield of lycopene by $429 \mu \mathrm{g} / \mathrm{g}$ and $1104 \mu \mathrm{g} / \mathrm{g}$, for cellulase and pectinase treated samples, respectively. Ultrasonic assisted technology for lycopene extraction from tomatoes was also reported by Eh et al. ${ }^{22}$, sonication enhanced the efficiency of relative lycopene yield. However, low temperature in ultrasonic assisted extraction minimises the degradation and isomerisation of lycopene, it attributes to the difficulty for the solvent to penetrate the compact tomato peel tissue and solubilize the pigment which is deeply embedded within the chromoplast membrane structures.
The objective of this work was to study the effect of several parameters related to solid-liquid extraction on lycopene recovery in tomatoes waste. Microwave assisted extraction was the selected technology. The conditions were optimised, with respect to degradation, isomeration and maximum recovery of lycopene. Furthermore, the stability of the isolated lycopene was carried out under different conditions in the aim to study the oxidative air degradation rate and the illumination effect.

\section{Experimental section}

\section{Raw material}

Fresh tomatoes waste (a mixture of skins and seeds), obtained from tomatoes processing company (located in northTunisia) in summer 2014, was used for the experiment. The material was ground with a mill $(0,1-0,5 \mathrm{~mm}$ particle size) after drying during 24 hours in hot air oven at $37^{\circ} \mathrm{C}$. Then it was stored in air tight containers until further use.

Prepared sample was investigated with three solid-phase extraction process: ultrasonic assisted extraction, microwave assisted extraction and homogenizer heating extraction.

\section{Chemicals}

All organic solvents used for extraction and UVVisible analysis were of analytical grade and purchased from Lab-Scan Ltd. (Dublin, Ireland). Solvents used for chromatography analysis were from HPLC grade and purchased from Sigma ( $\mathrm{St}$ Louis, MO). Lycopene standard (90-95\% purity) was purchased from Sigma-Aldrich Chemie Gmbh (Steinheim, Germany).

\section{Lycopene quantitative Analysis}

During preliminary tests of the extraction protocol (optimisation of the solid-tosolvent ratio, optimisation of the temperature and optimisation of the extraction time), an UV-Visible spectrophotometry (SHIMADZU/UV-1800) at 470 $\mathrm{nm}$, was used to quantify the amounts of total extracted lycopene.

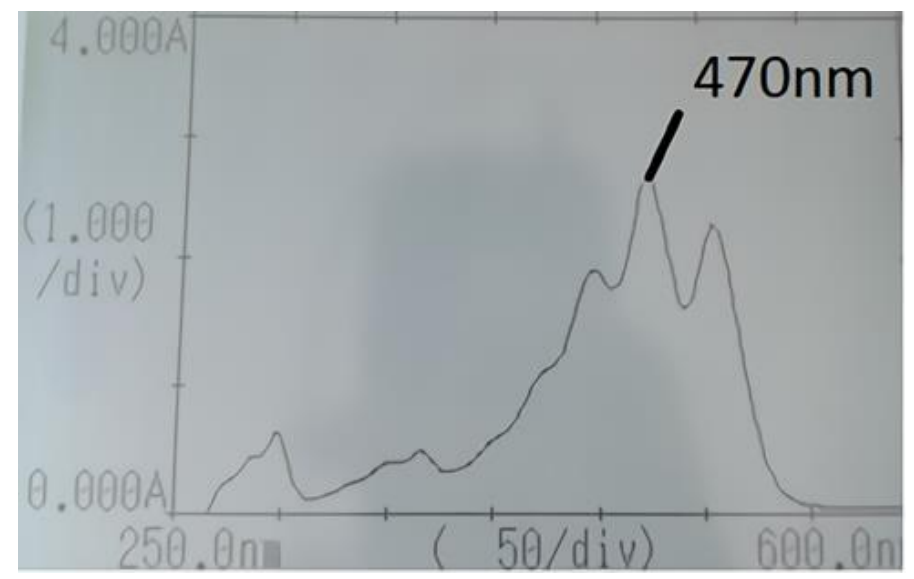

Figure 1. Absorption spectra of extracted lycopene in ethanol. 
Qualitative and quantitative analysis of extracted lycopene during UAE and MAE investigation were carried out by UPLC-DAD system consisted of UHPLC focused Utimate 3000 Dionex liquid chromatograph with DAD 3000 Dionex UV/Visible detector. The analysis was performed in triplicate injections, under the following conditions : reversed phase column: C18 Inertsil ODS-3 ; mobile phase: acetonitrile/methanol/ethylacetate (60:20:20); flow rate: $0,5 \mathrm{~mL} \cdot \mathrm{min}^{-1}$. The monitoring wavelength was: $470 \mathrm{~nm}$.

Lycopene was identified by comparing the retention time with that of the standard compound, which was also used to obtain calibration curve, in order to determine the total amounts of lycopene.

Afterwards, the above UPLC-DAD method was applied to investigate the stability assay for the extracted and vaccum dried lycopene.

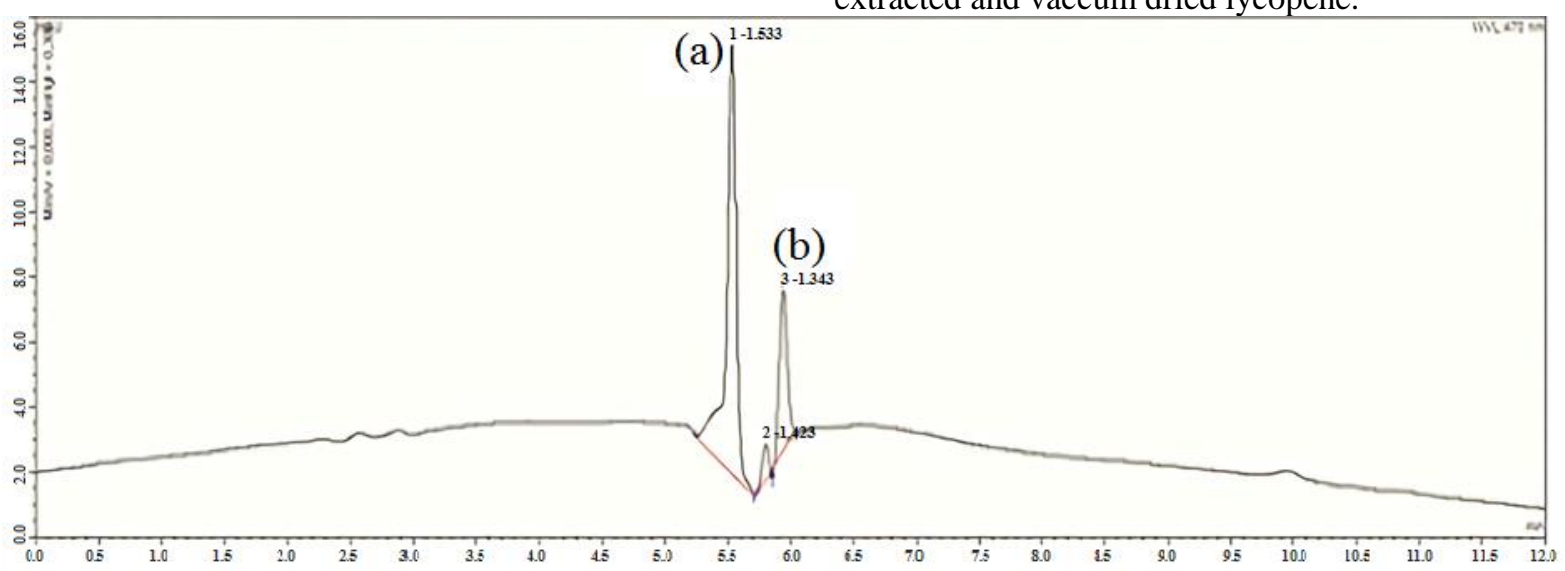

Figure 2. UPLC chromatogram of total lycopene (a) Z-lycopene (b) E-lycopene at $470 \mathrm{~nm}$, total concentration

\section{Experimental Section}

First of all, preliminary optimisation of the proper solvent, in term of its ability to dissolve lycopene was carried out. Homogenizer heating assisted extraction has been applied to determine the optimum solid-to-liquid ratio for the maximum lycopene release. Pur ethanol showed a high dissolve power, in addition to its advantages concerning its easy remove, its cost, its inertness and its low toxicity.

The combination of $1 \mathrm{~g}$ of the solid raw material into $10 \mathrm{~mL}$ of pur ethanol (1:10 solid-to -liquid ratio) results in an optimal ratio to be tested later for a maximum lycopene recovery with different assisted extraction techniques.

Furthermore, we studied the effects of the temperature $\left(25^{\circ} \mathrm{C}, 40^{\circ} \mathrm{C} ; 50^{\circ} \mathrm{C}\right.$ and $\left.60^{\circ} \mathrm{C}\right)$ and the extraction time ( $30 \mathrm{~min}, 60 \mathrm{~min}$ and $90 \mathrm{~min}$ ) on the solubility rate, and to ensure the complete diffusion of the target analyte from the matrix to the liquid phase, we performed an Homogenazer Heating Extraction (HHE). During the extraction operation, a simple sampling from the supernatant ethanolic extract was filtered with $0,45 \mu \mathrm{m}$ syringe filters and

\section{$0,7 \mu \mathrm{g} \cdot \mathrm{mL}^{-1}$.}

the amount of lycopene was determined in darkness by UV-Visible spectrophotometry.

Selected tempeature and time operating extraction using HHE protocol for the best recovery of lycopene applying Ultrasonic Assisted Extraction (UAE) assay carried out with an ultrasonic bath FLASC; $34 \mathrm{KHz}$ and for Microwave Assisted Extraction (MAE) assay using a MILESTONE, START D microwave.

All measurements realized for each tested process were performed three times.

\section{Conditions for stability study assay}

Recovered lycopene was vaccum dried into powder, then tested for degradation. Air oxidative degradation and light effects were separately studied: in clear glass container, amber glass container and air tight or open air container. Two conditionings were also prepared and tested for stability : the first one was lycopene in tablet, mixed with olive oil as a natural source of antioxidants and good lubricant, the second one was stored in a capsule. Both conditionings aimed to protect sample from light and air effects.

In total, six lots were stored in six different packing conditions at ambiant temperature as in Table 1.

Table 1. Lots for recovered lycopene powder stability assay.

\begin{tabular}{lcccccc}
\hline Lot & $(1)$ & $(2)$ & $(3)$ & $(4)$ & $(5)$ & Powder \\
\hline Form & Powder & Powder & Powder & Powder & $\begin{array}{c}\text { Compressed powder }+ \\
\text { olive oil }\end{array}$ & Amber/air tight \\
\hline Container & $\begin{array}{c}\text { Clear/air } \\
\text { tight }\end{array}$ & $\begin{array}{c}\text { Amber/air } \\
\text { tight }\end{array}$ & Clear & amber & Capsule \\
\hline
\end{tabular}




\section{Results and Discussions}

\section{Extraction process}

In the aim to control the rate of lycopene transfert into the extraction solvent by each process (HHE, UAE and MAE) and to compare the physical influence of each technology, the same conditions of temperaure, operating time and solidto-solvent ratio were fixed and used for each process. Preliminary testes result in the following conditions : the temperature was fixed at $45^{\circ} \mathrm{C}$, the extraction time at $30 \mathrm{~min}$ and the waste tomatoes sample-toethanol ratio at 1:10 (W/V). Lycopene recovery in each extract was measured by UPLC-DAD.

Highest yield was achieved by MAE process and the lower one by UAE. The ultrasonic forces enhancing lycopene diffusion into ethanolic phase seems leading to a possible degradation of the red pigment. Thus, for further optimisation studies of the extraction conditions, MAE process and HHE process would be peformed.
Effect of the temperature and the operating time on the lycopene recovery extraction

Another way of increasing the efficiency of the conventional extraction is to control the temperature effect during the extraction operation. Lycopene recovery was investigated using $\mathrm{HHE}$ process at: $25^{\circ} \mathrm{C} ; 40^{\circ} \mathrm{C} ; 50^{\circ} \mathrm{C}$ and $60^{\circ} \mathrm{C}$ within 30 min and 60 min time operating for each fixed temperature.

However, for the extraction of phyto-chemicals like lycopene from plants the use of elevated temperatures, generates undesirable reactions like the degradation of compounds (and loosing) which lose their biological activities ${ }^{23}$. Figure 1 shows that the best lycopene recovery is obtained at $50^{\circ} \mathrm{C}$, above this value, lycopene starts to lose its stability and a significant decrease of the absorbance is noted. When using $30 \mathrm{~min}$, as operating time extraction, we were able to dissolve the maximum of lycopene into ethanolic phase, while tempeature is less effective above $30 \mathrm{~min}$.

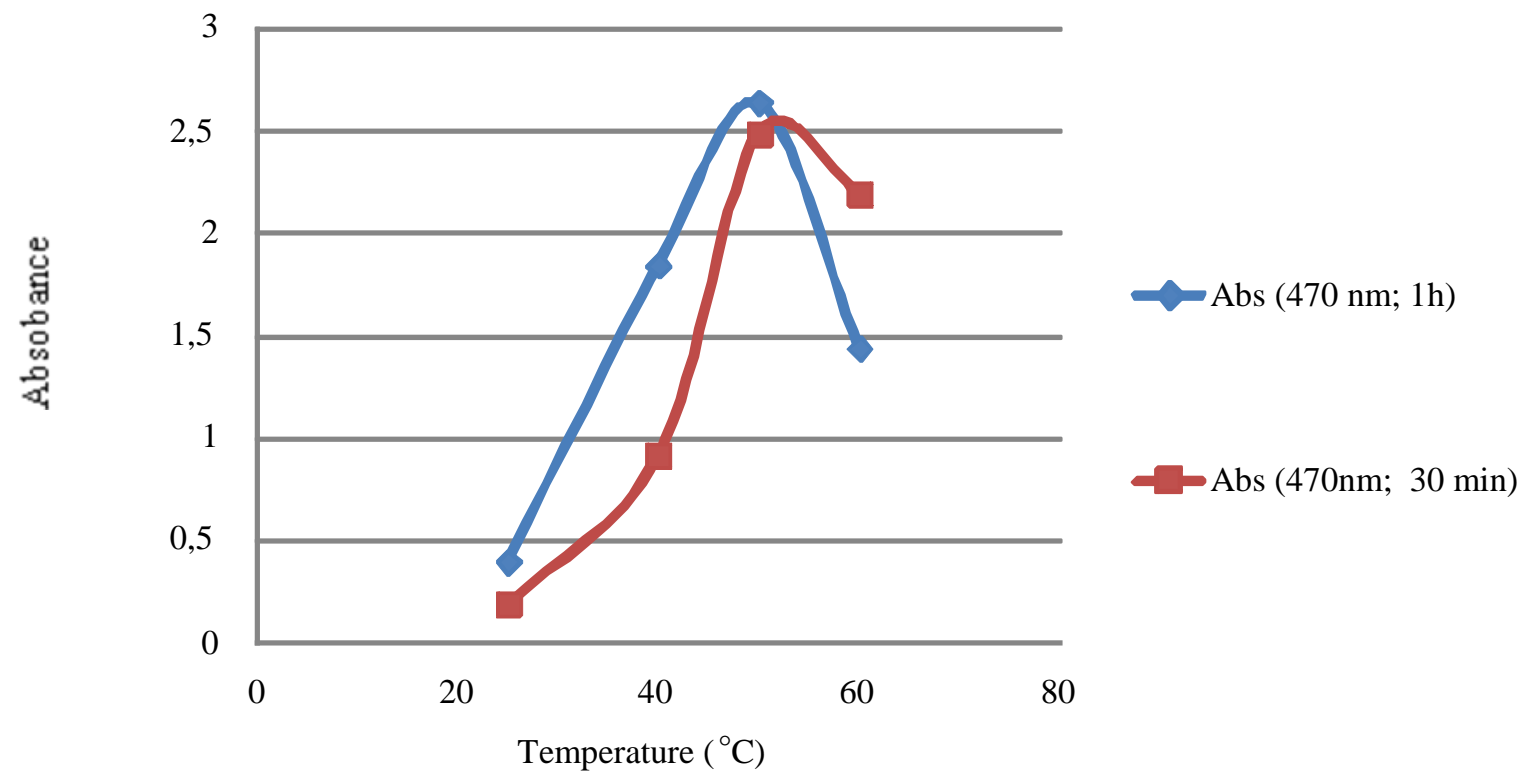

Figure 3. Effect of the temperature and the operating time on lycopene recovery by HHE process (Absorbance at $470 \mathrm{~nm})$.

\section{Optimisation of the MAE conditions}

Microwave irradiation involves disruption of hydrogen bonds, in addition of microwave-induced dipole rotation of molecules, and migration of ions. This mechanism enhances penetration of the solvent into the matrix, allowing the dissolution of lycopene to be extracted. MAE process was carried out, using the optimised operational time $(30 \mathrm{~min})$ and 1:10 $(\mathrm{W} / \mathrm{V})$, tomatoes waste:ethanol ratio. The effect of the temperature was re-examined, considering the effect of the pressure parameter during this process. Both $40^{\circ} \mathrm{C}$ and $50^{\circ} \mathrm{C}$, temperatures values were investigated at 20 bar and 30 bar microwave running pressure. Table 2 illustrates the UPLC-DAD quantification of lycopene yield improvment, which is induced by increase in pressure value. The pressure can bring up the temperature of the system and facilitates solvent penetration into tissues, and the solubilisation of the solute. Moreover, in closed vessels, the temperature can reach far above the boiling point of the solvent, leading to better extraction efficiency. Furthermore, the temperature is directly linked with the stability of the target molecule and our reached optimum $(5,9 \%)$ is selected at $50^{\circ} \mathrm{C}$ and 30 bar as optimised conditions for MAE process. 
Table 2. Effect of the temperature and the pressure on lycopene recovery by MAE process.

\begin{tabular}{lcccc}
\hline Temperature $\left({ }^{\circ} \mathrm{C}\right)$ & \multicolumn{3}{c}{40} & \multicolumn{3}{c}{50} \\
\hline Pressure (bar) & 20 & 30 & 20 & 30 \\
\hline Yield $(\%)$ & 4,1 & 4,6 & 5,1 & 5,9 \\
\hline
\end{tabular}

\section{Stability study of lycopene powder}

A low stability of lycopene is obtained due to light effect, which is the main cause of degradation. Regardless of the air tightening container storage, a significant degradation rate is detected when samples are exposed to day light. Lycopene is also undergoing a high oxidation when stored in open air container. Only slight changes were detected when we change open air container, by air tight container, whereas significant changes were found when we change clear container by amber one (Figure 4 and Figure 5).

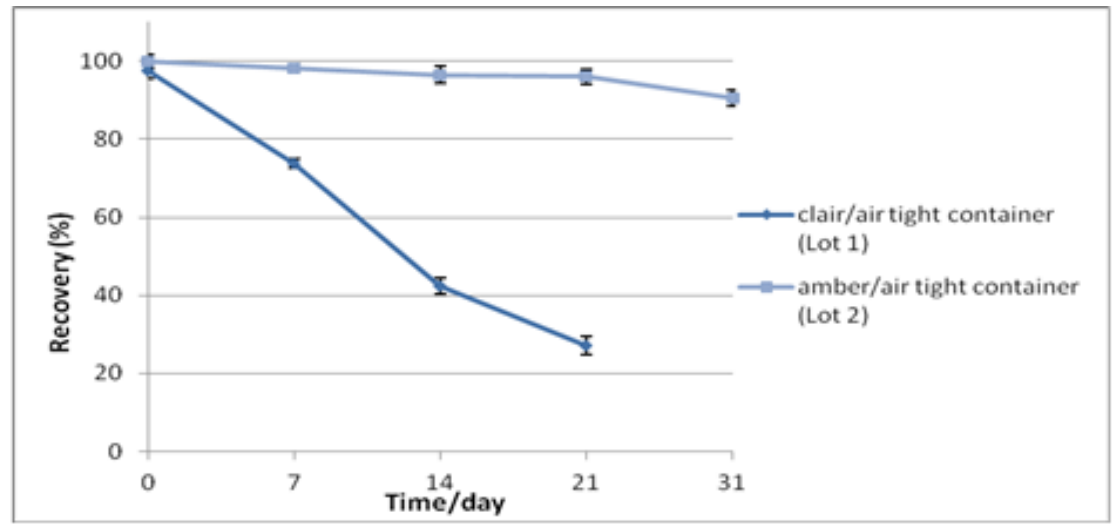

Figure 4. Degradation rate of the isolated lycopene powder for $\operatorname{Lot}(1)$ and Lot (2) $(\mathrm{SD}<2,9 \%)$.

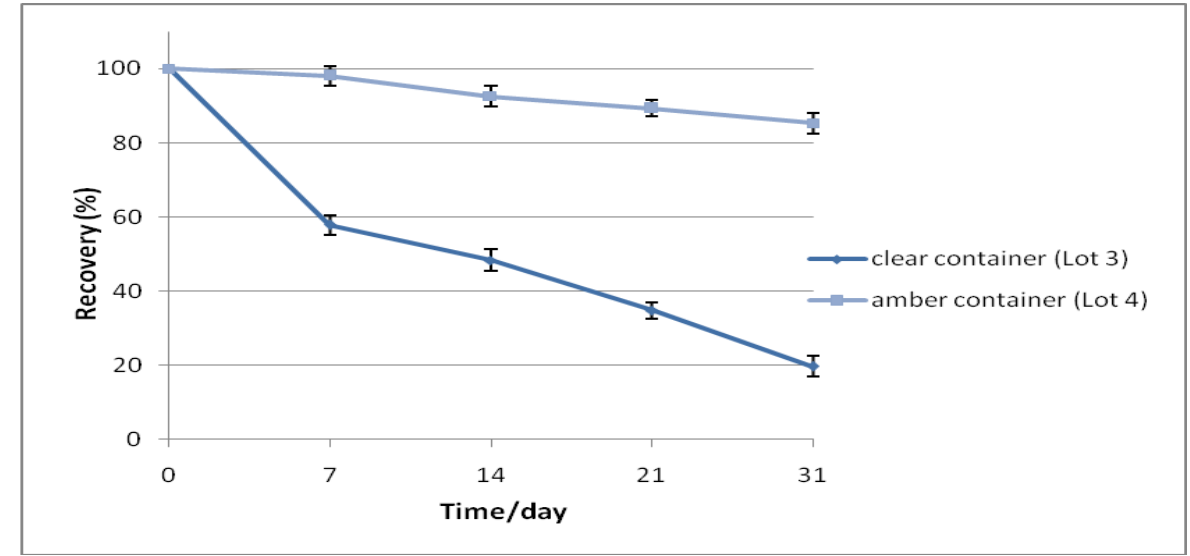

Figure 5. Degradation rate of the isolated lycopene powder for $\operatorname{Lot}(3)$ and $\operatorname{Lot}(4)(\mathrm{SD}<2,3)$.

For lot (5) and lot (6), the obtained results indicate that conditioning lycopene powder in capsule (is) as well as mixed with olive oil into tablet conditioning, protects the extracted sample and limits its photo-sensitivity and oxidative degradation (Figure 6).

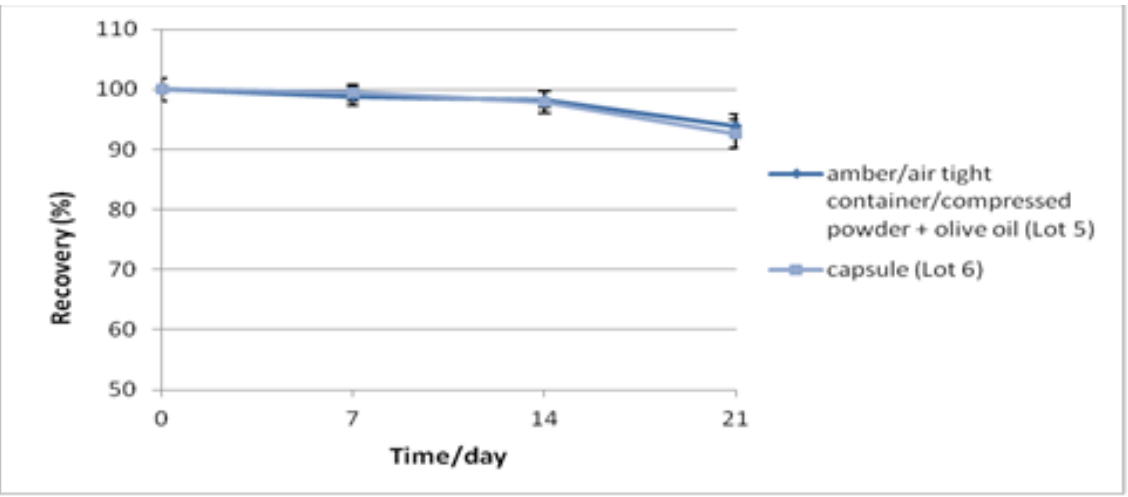

Figure 6. Degradation rate of the isolated lycopene powder for $\operatorname{Lot}(5)$ and $\operatorname{Lot}(6)(\mathrm{SD}<1,8 \%)$. 


\section{Conclusion}

The tomatoes waste processing could be a possible value-added product, in term of economic opportunity for the agri-food sector and viable solution to the disposal problem. The results of the present investigation prove that tomatoes waste represents an effective source for lycopene production.

Optimal conditions for the highest recovery extraction process were determined. Microwave assisted extraction process is selected, considering its advantages in terms of reduced extraction time, reduced solvent consumption, less environmental pollution and extraction yield improving to an average of $5,9 \mathrm{Wt}$. $\%$.

The stability of the extracted lycopene was investigated and shows a significant degradation when exposed to day light. Oxidative air degradation can be avoided by using an air tight container. Moreover, conditioning lycopene with olive oil as a natural antioxidants and lubricant for tablet form showed a promising combination for a novel benefit for bio-product development.

\section{References}

1- A. Schieber, F. C. Stintzing, R. Carle, Trends in Food Science and Technology, 2008, 12, 401413.

2- R. Luque, J. H. Clark, Sustainable Chemical Processes, 2013, 1, 10, 1-3.

3- I. MartínezValverde, M. J. Periago, G. Provan, A. Chesson, Journal of the Science of Food and Agriculture, 2002, 82, 323-330.

4- B. George, C. Kaur, D. S. Khurdiya, H. C. Kapoor, Food Chemistry, 2004, 84, 45-51.

5- S. K. Sharma, M. Le Maguer, Journal of Food Science, 1996, 2, 107-113.

6- R. K. Toor, G. P. Savage, Food Research, 2005, 38, 487-494.

7- V. Oreopoulou, C. Tzia, Utilization of ByProducts and Treatment of Waste in the Food Industry; Edidtion V; Springler Science \& Business Media; New York, 2007, 209-232.

8- K. W. Kong, H. E. Khoo, K. N. Prasad, A. Ismail, C. P. Tan, N. F. Rajab, Molecules, 2010, 15, 959-987.
9- P. Di Mascio, S. Kaiser, H. Sies, Archives of Biochemistry and Biophysics, 1989, 274, 532-538.

10- Q. Shixian, Y. Dai, Y. Kakuda, J. Shi, G. Mittal, D. Yeung, Food Reviews International, 2005, 21, 295-311.

11 - M. Kelkel, M. Schumacher, M. Dicato, M. Diederich, Free Radical Research, 2011, 45, 925-940.

12- G. Riccioni, B. Mancini, E. Di Ilio, T. Bucciarelli, N. D’Orazio, European Review for Medical and Pharmacological Sciences, 2008, 12, 183-190.

13- C. S. K. Lin, L. A. Pfaltzgraff, L. HerreroDavila, E. B. Mubofu, S. Abderrahim, J. H. Clark, A. Koutinas, N. Kopsahelis, K. Stamatelatou, F. Dickson, S. Thankappan, Z. Mohamed, R. Brocklesby, R. Luque, Energy Environmental Science, 2013, 6, 426-464.

14- I. F. Strati, V. Oreopoulou, International Journal of Food Science and Technology, 2011, 46, 23-29.

15- N. L. Rozzi, R. K. Singh, R. A. Vierling, B. A. Watkins, Journal of Agricultural and Food Chemistry, 2002, 50, 2638-2643.

16- E. Sabio, M. Lozano, V. Montero de Espinosa R. L. Mendes, A. P. Pereira, Industrial and Engineering Chemistry Research, 2003, 42, 6641-6646.

17- L. S. Kassama, J. Shi, G. S. Mitta, Separation and Purification Tehnology, 2008, 60, 278284.

18- U. Topal, M. Sasaki, M. Goto, K. Hayakawa, Journal of Agricultural and Food Chemistry, 2006, 54, 5604-5610.

19- B. P. Nobre, L. Gouveia, P. G. S. Matos, A. F. Cristiano, A. F. Palavra, R. L. Mends, Molecules, 2012, 17, 8397-8407.

20- S. M. Choudhari, L. Ananthanarayan, Food Chemistry, 2007, 102,77-81.

21- A. Zuorro, R. Lavecchia, F. Medici, L. Piga, Food and Bioprocess Technology, 2013, 6, 3499-3509.

22- A. L. S, Eh, S. G. Teoh, Ultrasonics Sonochemistry, 2012, 19, 151-159

23- F. Y. Du, X. H. Xiao, G. K. Li, Journal of Chromatography A, 2007, 1140, 56-62. 